\title{
Efficacy and safety of a new surgical method to treat malignant glaucoma in pseudophakia
}

T Żarnowski', A Wilkos-Kuc ${ }^{1}$, M Tulidowicz-Bielak' ${ }^{1}$, A Kalinowska', A Zadrożniak ${ }^{1}$ E Pyszniak ${ }^{1}$ and M Rękas ${ }^{2}$

\begin{abstract}
Purpose To assess the efficacy and safety of a relatively new surgical method in pseudophakic malignant glaucoma patients. Methods This study is a retrospective, noncomparative, interventional case series. Ten eyes of nine pseudophakic malignant glaucoma patients with mean age of 63.3 years were analysed. All 10 eyes underwent a novel surgical technique, an anterior chamber capsulo-hyaloidectomy and anterior vitrectomy through the peripheral iridectomy. Main outcome measures were: reformation of the anterior chamber, intraocular pressure (IOP), best-corrected visual acuity (BCVA), and complications.

Results All 10 eyes with pseudophakic malignant glaucoma were treated successfully by using a new surgical technique. All cases had a relief of aqueous misdirection with anterior chamber deepening during and after the surgery and post-operative intraocular pressure (IOP) normalization. No relapses have been observed so far. There were no complications during surgery and in the post-operative period.

Conclusion The presented surgical technique seems to be safe and effective in all cases of malignant glaucoma in pseudophakia.

Eye (2014) 28, 761-764; doi:10.1038/eye.2014.53; published online 14 March 2014
\end{abstract}

\section{Introduction}

The aim of malignant glaucoma treatment is to relieve the aqueous misdirection. Previous studies have reported limited efficacy of the treatment. Medical treatment induces posterior movement of the lens-iris diaphragm, reduces aqueous production, and shrinks the vitreous volume. Medical therapy alone is initially successful in $50 \%$ of eyes, however, the relapse rate is almost $100 \%$. In those cases that are refractory to medical and/or laser treatment, surgical treatment has to be used. ${ }^{1,2}$ The conventional vitrectomy and Nd:YAG laser capsulotomy with hyaloidotomy also have short-term effect with a relapse rate of up to $75 \% .^{3}$ Recently, some case reports or small case series suggest that combination of pars plana vitrectomy with zonulectomy and iridectomy might give promising results in pseudophakic eyes. ${ }^{4}$ In the light of our recent observations, it is questionable whether pars plana vitrectomy has to be performed to get fully successful results.

The aim of the present study is to evaluate the safety and efficacy of the simple technique that consists of anterior vitrectomy performed through the clear cornea, zonulectomy, peripheral capsulectomy, and iridectomy (if not done previously) in pseudophakic malignant glaucoma.

\section{Materials and methods}

Ten pseudophakic patients with mean age of $63.3 \pm 12.5$ years were treated for malignant glaucoma in the Department of Diagnostics and Microsurgery of Glaucoma, Medical University, Lublin, Poland in 2012-2013. The diagnosis of malignant glaucoma was based on shallow or flat anterior chamber and elevated IOP, with the presence of patent iridotomy and normal posterior segment (absence of suprachoroidal effusion or haemorrhage). All patients had one or more risk factors for developing malignant glaucoma (history of primary angle closure, hyperopia, excessive filtration after trabeculectomy, or history of malignant glaucoma in neighbouring eye). All patients had a history of primary angle closure and eight
${ }^{1}$ Department of Diagnostics and Microsurgery of Glaucoma, Medical University of Lublin, Lublin, Poland

${ }^{2}$ Department of Ophthalmology, Military Institute of Medicine, Warsaw, Poland

Correspondence: T Żarnowski, Department of Diagnostics and Microsurgery of Glaucoma, Medical University of Lublin, Chmielna 1, Lublin, 20-079, Poland Tel/Fax: + 48815324827 E-mail: zarnowskit@ poczta.onet.pl

Received: 19 July 2013 Accepted in revised form: 5 February 2014 Published online: 14 March 2014 
Table 1 Summary of the patients' data

\begin{tabular}{|c|c|c|c|c|c|c|c|c|}
\hline \multicolumn{5}{|c|}{ Demographic data } & \multicolumn{2}{|c|}{$I O P(m m H g)$} & \multicolumn{2}{|c|}{$C D V A(\log M A R)$} \\
\hline Age/gender & Eye & $A X L$ & History & Time (months) & Pre-op & 12 months & Pre-op & 12 months \\
\hline $61 ㅇ$ & $\mathrm{~L}$ & 20.58 & PACG, FT & 12 & 60 & 14 & 1.70 & 1.70 \\
\hline $63 ㅇ$ & $\mathrm{R}$ & 20.85 & PACG, F & 18 & 42 & 18 & 0.30 & 0.10 \\
\hline 809 & $\mathrm{R}$ & 20.15 & PACG, F & 12 & 32 & 20 & 2.40 & 2.40 \\
\hline 769 & $\mathrm{R}$ & 21.25 & PACG, T & 12 & 34 & 16 & 0.49 & 0.40 \\
\hline $400^{\pi}$ & $\mathrm{R}$ & 21.69 & PACG, T & 12 & 42 & 13 & 0.30 & 0.15 \\
\hline 61 & $\mathrm{R}$ & 20.49 & PACG, PEX, FT & 18 & 38 & 15 & 1.70 & 2.40 \\
\hline $61 ㅇ$ & $\mathrm{~L}$ & 22.31 & PACG, FT & 12 & 23 & 6 & 0.52 & 0.40 \\
\hline 80 & $\mathrm{~L}$ & 21.07 & PACG, PEX, T & 6 & 40 & 18 & 1.0 & 0.40 \\
\hline 58 & $\mathrm{~L}$ & 22.25 & PACG, F & 12 & 28 & 16 & 0.40 & 0.60 \\
\hline $53 ㅇ$ & $\mathrm{~L}$ & 21.34 & PACG, T & 12 & 38 & 12 & 1.10 & 0.30 \\
\hline
\end{tabular}

Abbreviations: AXL, axial length; CDVA (log MAR), corrected distance visual acuity; F, phaco; FT, phacotrabeculectomy; IOP, intraocular pressure; PACG, primary angle closure glaucoma; PEX, pseudoexfoliation syndrome; R/L, right/left eye; T, trabeculectomy.

eyes were hyperopic (mean $\mathrm{AXL}=21.30 \pm 1.06 \mathrm{~mm}$ ). Three patients had a history of malignant glaucoma in the fellow eye. In all our patients malignant glaucoma occurred after surgery: phacoemulsification (three eyes), phacotrabeculectomy (three eyes), and trabeculectomy (four eyes) (Table 1).

\section{Surgical technique}

All surgeries were performed by the same anterior segment surgeon (TZ). In all cases peribulbar anaesthesia was used. Temporal clear corneal incision was made with 1.2 sideport knife. With the same knife clear corneal incision was performed at 12 o'clock position. The incision was created as to penetrate through peripheral iris (or centre of the iridectomy done previously), zonules, and the margin of the periphery of the capsular bag. Through the pupil, the tip of the knife was observed in the microscope, in the vitreous cavity, centrally just behind the posterior capsule. Twenty-gauge vitreous cutter (Infiniti, Alcon, Fort Worth, TX, USA) was introduced through newly created canal to the same place behind the bag. Several cycles of the cutter were performed and some deepening of the chamber was observed. As soon as the anterior chamber became deeper, irrigating cannula connected to the bottle with Ringer solution was introduced to the anterior chamber. The height of the bottle was adjusted as to obtain moderate IOP (20-30 $\mathrm{mm} \mathrm{Hg})$. While cutting anterior vitreous, anterior hyaloid, zonules, and the periphery of the bag, vitrectome was slowly being withdrawn finally reaching the iris as to enlarge peripheral iridectomy (if necessary) to $\sim 2 \mathrm{~mm}$. Vitrectomy was discontinued while substantial deepening of the anterior chamber was observed. To make sure that all vitreous was completely removed from the iris opening and the wound, the small amount of triamcinolone solution was administered to the anterior chamber. While chamber was becoming deeper the range of anterior synechiae was usually easily discernible. Some synechiae broke spontaneously on deepening, the remaining were broken with the Sinskey hook with the use of goniolens. As there was chance to restore the function of filtering fistula, the surgery was performed as not to handle or damage the bleb. Finally, the 12 o'clock corneal incision was closed with the 10.0 nylon suture and temporal wound with the corneal hydratation (Figure 1).

\section{Results}

All patients with aqueous misdirection syndrome had relief of the aqueous misdirection post-operatively with anterior chamber deepening and IOP lowering. None of the patient required atropine to keep anterior chamber deep.

Mean preoperative IOP was $37.7 \pm 9.9 \mathrm{~mm} \mathrm{Hg}$ and was reduced to $13.5 \pm 2.7$ at the end of follow-up $\left(\chi_{\text {ANOVA }}^{2}=31.16 ; P=0.00002\right.$; range means $=3.75$, range sum $=30.0 ; P<0.05)$. Mean CDVA (log MAR) improved from $0.99 \pm 0.73$ to $0.88 \pm 0.91$ at the end of follow-up (paired Wilcoxon's test, $P=0.363$ ). Improvement of 1 Snellen line charts occurred in $50 \%$ of eyes, CDVA did not change in $40 \%$ of the eyes, deterioration of 1 or more lines were determined in $10 \%$ of the operated eyes. No complications were observed either during or after the surgery (eg bleeding, macular oedema, and peripheral retinal tears).

None of the 10 eyes had any recurrence during follow-up, with a mean duration of $12.0 \pm 4.0$ months (range, 6-18.0 months; Table 1).

\section{Discussion}

Pars plana vitrectomy has been thought to prevent aqueous accumulation inside the vitreous cavity and it 

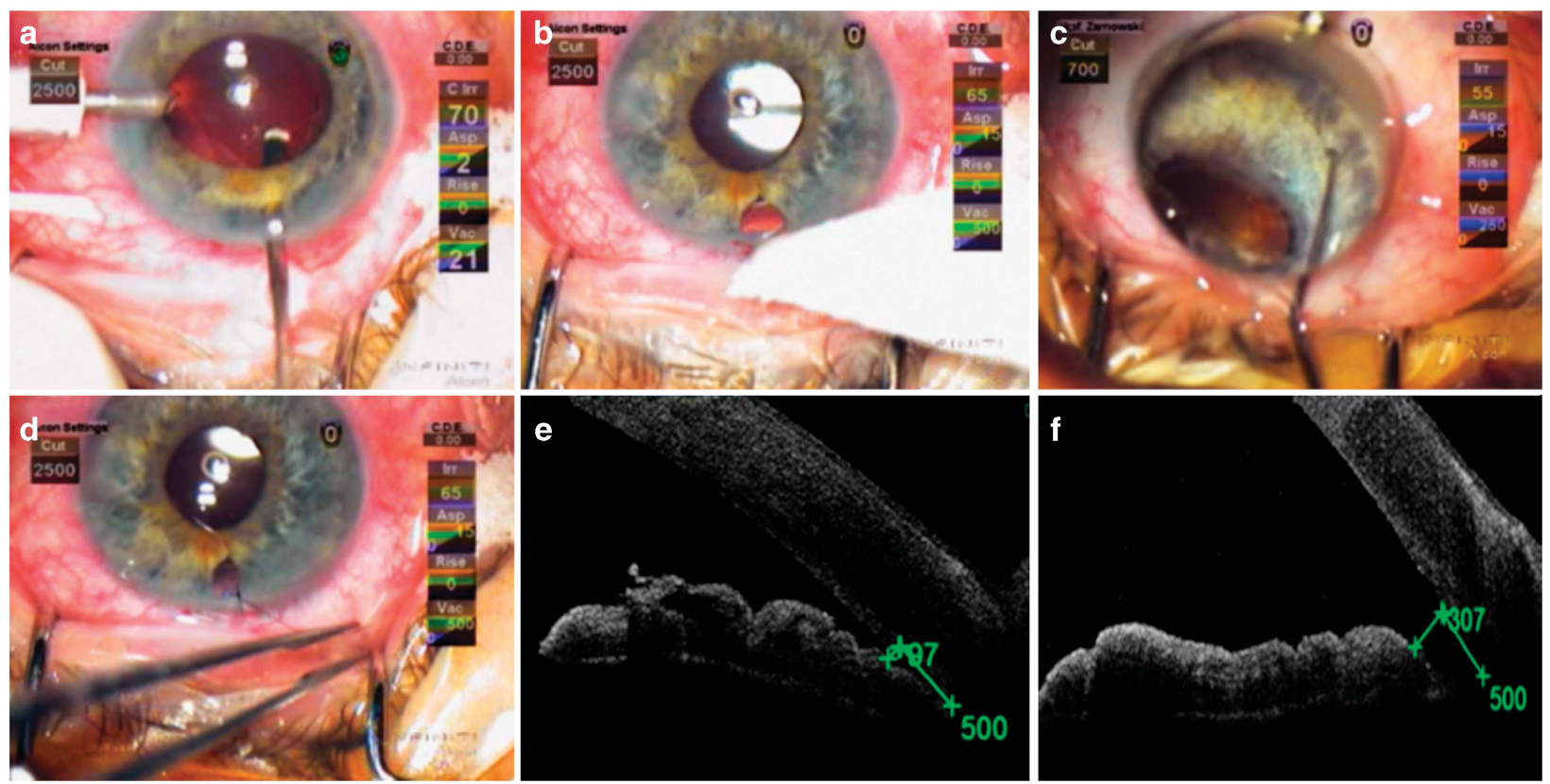

Figure 1 (a) Twenty-gauge vitreous cutter introduced through newly created canal to the place behind the posterior capsule, AC maintainer introduced to the anterior chamber through sideport. (b) Enlarged peripheral iridectomy to $\sim 2 \mathrm{~mm}$ with the use of vitrectome. (c) Synechiae broken with the Sinskey hook with the use of goniolens. (d) Corneal incision closed with the 10.0 nylon suture. (e) Patient with malignant glaucoma before surgery. Low level of AOD500 (97 $\mu \mathrm{m})$ suggests closure of the angle. (f) Patient after anterior chamber capsulo-hyaloidectomy and anterior vitrectomy through the peripheral iridectomy. Following the procedure, AOD500 increased to $307 \mu \mathrm{m}$. The AOD500 was defined as the linear distance, perpendicular to the trabecular meshwork, measured between the point of the inner corneoscleral wall (which was $500 \mu \mathrm{m}$ anterior to the scleral spur) and the iris.

has been reported to treat pseudophakic malignant glaucoma. ${ }^{1,5}$ However, this may not be enough to disrupt the cycle of malignant glaucoma because it was postulated that all of the tissues (iris, lens capsule, zonule, and anterior vitreous) had to be removed to create a permanent passage between the anterior chamber and the vitreous cavity. ${ }^{5,6}$ Part of the problem is that, during conventional vitrectomy peripheral vitreous can hardly be completely removed, and that is why relapse rate may be very high. Debrouwere et al. ${ }^{5}$ emphasized that only total vitrectomy was effective in $100 \%$ of patients on condition it is combined with zonulectomy, iridectomy, and capsulectomy. However, this attitude requires two accesses; it is time consuming, does not save the conjunctiva, and is prone to potential complications.

In our case series, all the 10 cases of pseudophakic malignant glaucoma patients were treated successfully using an anterior chamber capsulo-hyaloidectomy and anterior vitrectomy through the peripheral iridectomy. In all the cases we observed significant deepening of the anterior chamber and elimination of aqueous blockade during surgery. The procedure is fast, simple, and easily accomplished by an anterior chamber surgeon. It seems that if the passage between the anterior chamber and vitreous cavity is established and maintained, the procedure is effective without the need of total vitrectomy. One should keep in mind that the aim of the surgery was to restore the anterior chamber, thus, in chronic glaucoma effort must be done to maintain patency of filtration fistula. Therefore, soon after the malignant glaucoma is diagnosed the procedure should be performed without delay to salvage the bleb.

Because of the relative paucity of pseudophakic malignant glaucoma cases in one centre, it seems that multicentre studies are needed to establish long-term success and optimal instrumentation (eg 20 vs 23 gauge).

\section{Summary}

\section{What was known before}

- Surgical treatment of malignant glaucoma has limited success, and even total vitrectomy does not warrant success with high rate of relapses.

What this study adds

- This study presents the technique of anterior hyaloidectomy combined with peripheral iridectomy, zonulectomy, and peripheral capsulectomy that so far offers $100 \%$ success rate to restore anterior chamber with no relapses and no complications.

- It seems that further multicentre studies are needed to establish long-term success and optimal instrumentation in larger group of patients. 


\section{Conflict of interest}

The authors declare no conflict of interest.

\section{References}

1 Shahid H, Salmon JF. Malignant glaucoma: a review of the modern literature. J Ophthalmol 2012; 2012: 852659.

2 Harbour JW, Rubsamen PE, Palmberg P. Pars plana vitrectomy in the management of phakic and pseudophakic malignant glaucoma. Arch Ophthalmol 1996; 114: 1073-1078.

3 Little BC, Hitchings RA. Pseudophakic malignant glaucoma: NdYAG capsulotomy as a primary treatment. Eye 1993; 7: 102-104.
4 Pasaoglu IB, Altan C, Bayraktar S, Satana B, Basarir B Surgical management of pseudphakic malignant glaucoma via anterior segment-peripheral iridectomy capsule-hyaloidectomy and anterior vitrectomy. Case Rep Ophthalmol Med 2012; 2012: 794938. doi:10.1155/ 2012/794938.

5 Debrouwere V, Stalmans P, Van Calster J, Spileers W, Zeyen T, Stalmans I. Outcomes of different management options for malignant glaucoma: a retrospective study. Graefes Arch Clin Exp Ophthalmol 2012; 250: 131-141.

6 Bitrian E, Caprioli J. Pars plana vitrectomy, hyaloidozonulectomy and iridectomy for aqueous humor misdirection. Am I Ophthalmol 2010; 150: 82-87. 